$\star$ Rhizomes: Cultural Studies in Emerging Knowledge: Issue 32 (2017)

\title{
The Other/ness Media Machine
}

\author{
Review by Craig Saper
}

Gloria Sutton, The Experience Machine: Stan VanDerBeek's Movie-Drome and Expanded Cinema. Cambridge, MA: MIT Press 2016.

Stan VanDerBeek has had a well-known and lasting influence on experimental animation and filmmaking as well as happening-like interactive artworks. Best known of all of his innovations, the "movie-drome," required spectators to enter a domed building, inspired by Buckminster Fuller's geodesic domes, lie on the floor, and watch many often abstract and psychedelic animated films projected on the inside of the dome using multiple projectors. Although he started making films for the movie-drome as early as 1957, and built the first one in 1963, he did not fully realize the cosmic potential of the experience until the late 1960s and 70s, when participants would often heighten the experience with hallucinogenic drugs and he called the projections a "Newsreel of Dreams" and "World Wide Image Libraries" (like an imagined internet projection with a feedback loop from those in one drome to all the other dromes in "a culture de-compression chamber" or "culture-inter-com"). VanDerBeek's work has recently had a posthumous renaissance with its prominent inclusion in major museum exhibitions, and also with the publication of Gloria Sutton's 2016 book reviewed here. This renewed interest is obviously due in part to his work projecting directly on our era of Internet image banks and surreal news assemblages much like Marshall McLuhan has since the early 1990s gained increasing legitimacy as the sage of the Internet's global village. Although VanDerBeek never dropped out of the picture, he was just in a category marginal to both histories of art, cinema, animation, or psychedelic techno counter-cultures. He imagined that his drome's feedbacks would communicate like "extraterrestrial whales" and send messages through satellite signals at the speed of light using un-invented optics. In retrospect nearly 50 years later, his experiments serve as precursors to experiments in e-media art and also now mundane gadgets like iPads and other tablets; in a video now online, Stan demonstrates a small flat screen tablet that you can write on directly in order to draw pictures or finger paint -- nothing extraordinary today, but the video was made in the early 1970s. One area less well known even to those who study VanDerBeek's works, and which Sutton discusses, is VanDerBeek's inclusion in the experimental poetry scene in the 1960s and 70s.

To write a review of Sutton's The Experience Machine, which chronicles only half of Stan's groundbreaking cornucopia of works through 1969, one needs a certain perspective on those works, and it is not a proscenium view of the work or the book. One needs a more visceral and bodily experience to truly appreciate both this relatively new art historical biography and the history of the Movie-Drome. One needs a personal or peculiar experience, perspective, and multimedia subjectivity. When I moved to Baltimore in 2011, a real estate advertisement caught my eye. It was for Stan VanDerBeek's former house in Relay, Maryland close to the university where he taught in the 1980s. There were pictures of the house and the property. One picture showed an amorphous shed-like building. The experimental animator, Lynn Tomlinson, and I immediately recognized the building as a "movie-drome" viewing dome. The caption under that picture nicely, and unwittingly, illuminates VanDerBeek's career, and the reception of his work in expanded cinema: the caption read "Other." Although we rushed there as soon as we arrived, the new owners had torn the "other" building down before we could see it. The university where he taught also figuratively tore down VanDerBeek by marginalizing him to less than a footnote in that university's 50-year celebration histories and in a series of discussions and interviews with those who followed him in building the university's art and "intermedia" MFA program. His work, and unconventional methods, were 
too far-out of the neoliberal mainstream to be celebrated by any institution, and his truly foundational intermedial work did not fit neatly in the given historical categories of art, art movements, experimental animation or media, or even the non-disciplinary visual poetry. His rightly suspicious heirs also make writing about Stan challenging as one asked me not to record my interview with her. All this friction about his legacy, makes him more interesting, if still "difficult" and "other" -- maybe because of, rather than in spite of, his occupying the "other" category that was never quite recuperated, and not easily projected on to the white walls of a museum instead of on the ceiling of a movie-drome with an audience on an involutionary trip. The very possibility of our immersive Internet culture, its endless collaged-stream of glocal otherness and misfits, is also what has kept VanDerBeek outside the wall of academic legitimation. The university where he taught has not yet built the VanDerBeek intermedia-drome, but that is in part because no one had written the history of his experiments as a blue-print for future possibilities of innovation.

Sutton's book enters my peculiar view with this historical context in mind, and it might reflect a readiness to salvage the experience machine with all of its prescience of our mundane technologies, but made strange again as seen through the drome-lens. Sutton not only takes into account the full breadth of VanDerBeek's media projects, including archival materials about unrealized or forgotten projects, but also shifts the discussion to a poetics of experience and situations - a shift that l've been chronicling from concrete and visual poetry through experimental animation and media to new sociopoetic projects like networked art. The chapter on VanDerBeek's work on Poemfields (1964-1969), the digitally produced text/image/sound animations (sometimes projected in a MovieDrome) places his work in relation to other poetry experiments at the time. The Poemfields "melded the syntax of concrete poetry with the programming mechanics of early computing to generate a new type of animation that presented poetry in cinematic time" (Sutton, 16), and in doing so, it reoriented poetry toward the asemic, situational, and experiential. It opened an emerging knowledge in the areas of composition, creative writing, and literary studies - which now propels a minority-rhetoric of electracy and craft-literacy.

That consideration of the work as poetry leading to a experience-, or socio-, poetics will prove particularly fertile ground for those scholars and poets involved in electronic literature and the organizations that look to combine these areas like ELO, HASTAC, and SLSA, if not SIGGRAPH, MLA, CAA, and SCMS. In The Experience Machine, VanDerBeek's works make sense in terms of the Fluxus projects with which he was associated, and, more importantly, open the door to thinking of "designed experiences" not just medium-specific (or multimedia) projections. Sutton's copious unpacking of the archive suggests an effort to shift subjectivity through "a transformation of the spectator" (Sutton, 187), and gives me the tools to consider the work as an effort to build academic programs for emerging knowledge and outside of the disciplinary boundaries, and object of study constrained foci in art, computer technology, media, psychology, and poetics programs.

Although the book concludes, correctly, in 1969, when VanDerBeek shifted away from the groundbreaking, and now newly relevant, media-poetry and movie-drome, he went on to do important collaborations, work in new technologies, and teach and research in a university. His project with Joan Brigham projected images on to steam, in the mid- to late 1970s, to once again disrupt the neat proscenium framed relationship between artist and audience. Now, he was increasingly interested in inserting the ephemerality of dreams onto public spaces; it was an analogy for thinking of a larger politics of making the intimate and unconscious dreamworld in public apparitions. This involutionary work has not been exceeded in any of the current immersive media experiments, but it still serves as a precursor and a future goal. The audience quite literally changed the image, and the steamprojection changed the tactile view and experience. When he taught at the university, he also wanted students to study/create "Dream Media Research," and that type of STEAM research now seeps around the halls of academia even if ephemerally, emerging, and fleetingly. Gloria Sutton's book will create a remarkably useful door for students to enter even if her book describes clearly and copiously the workings of the experience machine through various iterations rather than demonstrate its projective possibilities. 


\section{Cite this Review}

https://doi.org/10.20415/rhiz/032.r04

RHIZOMES ISSN 1555-9998 $\star 230$ East Hall Bowling Green State University Bowling Green, OH 43403

Editors: Ellen Berry and Carol Siegel. Reviews editor: Craig J. Saper. Technical editor: Helen J Burgess 